\title{
ARTICLE
}

\section{Immune abnormalities across psychiatric disorders: clinical relevance}

\author{
Valeria Mondelli, Paola Dazzan \& Carmine M. Pariante
}

\begin{abstract}
Valeria Mondelli is a senior clinical lecturer at the Institute of Psychiatry, Psychology \& Neuroscience (loPPN), King's College London. Her main research interest is the role of stress and of biological systems involved in the stress response in the pathogenesis of psychiatric disorders and in the interplay between physical and mental health. Paola Dazzan is a reader in the neurobiology of psychosis at the IoPPN. She is internationally known for her translational work on biological risk factors and imaging in the early stages of psychosis. Carmine M. Pariante is Professor of Biological Psychiatry and Head of Sections for the Stress, Psychiatry and Immunology Laboratory and Perinatal Psychiatry at the loPPN. He also leads one of the world's largest research groups dedicated to the study of the biological mechanisms through which stress affects mental health using both basic and clinical research experimental designs.

Correspondence Dr Valeria

Mondelli, Sections of Perinatal Psychiatry \& Stress, Psychiatry and Immunology (SPI-Lab), The James Black Centre, Institute of Psychiatry Psychology \& Neuroscience, King's College London, 125 Coldharbour Lane, London SE5 9NU, UK. Email: valeria.mondelli@kcl.ac.uk
\end{abstract}

\begin{abstract}
SUMMARY
It is well established that the immune system can modulate brain functioning and influence behavioural processes. Awareness of communication between the immune and nervous systems has, over the years, progressively heightened interest in the relationship between psychiatric disorders and immune function. By reviewing findings from studies investigating inflammation in the periphery and in the central nervous systems, we summarise here the evidence linking inflammation to the development of depression, schizophrenia and bipolar disorder. We discuss how a pathophysiological role for inflammation has now been recognised across different psychiatric disorders, at least in a significant subpopulation of patients. Finally, we discuss a possible role for these findings in the development of future diagnostic classifications of psychiatric disorders as well as of new treatment strategies.
\end{abstract}

\section{LEARNING OBJECTIVES}

- Increase knowledge of the immune system and inflammatory markers

- Learn about the most recent research findings linking inflammation to onset of psychiatric disorders

- Understand the clinical relevance of increased inflammation across different psychiatric disorders, in particular in the context of the potential for future therapeutic strategies

\section{DECLARATION OF INTEREST}

None

Over the years, we have become increasingly aware that the brain is only one part of the complex machine behind the onset of psychiatric disorders. Consequently, if we want to understand more about the pathogenesis of these disorders, we cannot separate the brain from what is happening in the rest of the body. Indeed, this seems to be true if we consider the immune system. It is now well established that the immune system can modulate brain functioning and influence behavioural processes. This modulation can be mediated by peripheral inflammatory signals to the brain as well as by central inflammatory processes produced within the brain (as in the case of neuroglial communication) (Yirmiya 2011).

The immune system is the primary biological system providing the initial line of defence against infectious agents and various forms of injury. Some of the evidence that supports the role of the immune system in the onset of psychiatric disorders comes from the observation of striking similarities between the symptoms of cytokineinduced sickness behaviour and depression (Dantzer 2008). Cytokine-induced sickness behaviour refers to the dramatic behavioural changes that take place when people experience a viral or bacterial infection. People experiencing an infection often feel feverish and nauseated, ignore food and beverages, and lose interest in their physical and social environments. They become easily tired, have problems with their sleep, feel depressed and irritable, and can experience mild cognitive disorders (Dantzer 2008). Furthermore, over the past few years, it has been established that pro-inflammatory cytokines can induce true major depressive disorders in physically ill patients with no previous history of mental illness.

This emerging evidence has led to an increased research interest in the relationship between psychiatric disorders and immune function. Although perhaps most prominent in depressive disorders, evidence suggesting the presence of inflammation has been reported in a broad range of psychiatric disorders. Inflammation in this context refers to systemic, chronic, low-grade inflammation, which is different from what we observe in response to acute infections, because (a) it is systemic and not limited to a local site of injury or infection, (b) it is of lesser magnitude than other types of inflammation, (c) it is typically a longer-term phenomenon, as opposed to the transient nature of inflammatory responses to infection, and (d) it does not have a clear origin or does not follow an apparent stimulus, such as infection or injury (Rohleder 2014). 
In this article, we give an overview of the evidence supporting the presence of low-grade inflammation across different psychiatric disorders, and discuss its potential clinical relevance. In particular, we are going to focus on depression, psychosis and bipolar disorder, as these are the disorders on which most of the research in this area has been conducted so far.

\section{Evidence of peripheral inflammation in psychiatric disorders}

To date, measurement of levels of inflammatory markers in the blood has been the most used research approach to investigate the presence of inflammation in psychiatric disorders. This is possibly due to the ease with which samples can be obtained, compared with investigating inflammation directly in the brain. As a result, most of the evidence supporting increased activation of the immune system in psychiatric disorders comes from these studies. Results are summarised in Box 1 and Table 1 and discussed in more detail below.

\section{Cytokines}

Cytokines are certainly the main inflammatory markers that have been investigated in these studies. Cytokines are low molecular weight proteins that act as key messengers of the immune system. They coordinate the immune response both in the periphery of the body and in the brain. Cytokines are also thought to be important mediators of the 'cross-talk' between the central nervous system and the immune system (Upthegrove 2014). Research has consistently reported abnormalities in cytokine levels in several psychiatric disorders, although with differences across diagnoses as well as a degree of inconsistency within single disorders (Baumeister 2014).

\section{Cytokines and depression}

In depression, the most consistent finding is the presence of elevated levels of interleukin-6 (IL-6), a pro-inflammatory cytokine capable of crossing the blood-brain barrier (Howren 2009; Dowlati 2010; Hiles 2012; Liu 2012). Similarly, tumour necrosis factor alpha (TNF- $\alpha$ ), a pro-inflammatory cytokine mainly involved in the regulation of immune cells, has also been reported as significantly elevated in depressed patients (Dowlati 2010; Liu 2012). In contrast, the evidence for IL-1 $\beta$, which is involved in the activation of several key inflammatory pathways, remains more inconclusive. In particular, one meta-analysis did not find significant differences (Dowlati 2010),
BOX 1 Association of peripheral C-reactive protein (CRP) levels with psychiatric disorders

- CRP levels have been reported consistently elevated in schizophrenia and in bipolar disorder

- Experience of childhood maltreatment has been found to be associated with high CRP levels in both depression and schizophrenia

- CRP levels are inversely correlated with cognitive performance in patients with bipolar disorder

another found a significant elevation of IL-1 $\beta$ with an effect that appeared to be dose-dependent according to clinical status (Howren 2009), and yet another found a similar elevation only in European samples (Liu 2012). Other interesting, although less replicated, findings show increased levels of IL-1 receptor antagonist (IL-1RA), a nonproductive ligand on IL-1 receptors, which may be considered as anti-inflammatory owing to its inhibition of IL-1 $\beta$ effects (Howren 2009). Furthermore, depressed patients also show increased levels of soluble IL-2 receptor (sIL-2R) (Liu 2012), which primarily acts as a storage reservoir for circulating IL-2 in order to increase longevity of IL-2 signalling (Yang 2011). Interestingly, studies have as yet failed to find any significant difference in IL-2 levels in depressed patients (Dowlati 2010; Liu 2012). However, this might be related to the limitations of laboratory analysis methods, which in the case of elevated levels of sIL-2R/IL-2 may fail to identify the bound IL-2 accurately (Baumeister 2014).

\section{Cytokines and schizophrenia}

In schizophrenia, the most consistent finding appears again to be an elevation of IL- 6 levels, similar to what has been reported in depression (Potvin 2008; Miller 2011; Mondelli 2011; Di Nicola 2013). Furthermore, significant elevation of sIL-2R is also reported quite consistently in patients with schizophrenia (Potvin 2008; Miller

\section{TABLE 1 Cytokines associated with various psychiatric disorders}

\begin{tabular}{|c|c|c|c|}
\hline \multirow[b]{2}{*}{ Disorder } & \multicolumn{3}{|c|}{ Evidence of association } \\
\hline & Strong & Moderate & Inconclusive \\
\hline Depression & IL-6, TNF- $\alpha$ & & IL-1 $\beta, I L-1 R A$, sIL-2R \\
\hline Schizophrenia & $\mathrm{IL}-6$ & IL-1RA, IL-12 & IL-1 $\beta$, TNF- $\alpha$, IFN- $\gamma$ \\
\hline Bipolar disorder & $\begin{array}{l}\text { IL-4, slL-2R, sIL-6R, } \\
\text { TNF- } \alpha, \text { sTNFR1, }\end{array}$ & & IL-1 $\beta$, IL-1RA, IL-10 \\
\hline
\end{tabular}

IFN, interferon; IL, interleukin; RA, receptor agonist; s...R, soluble receptor; TNF, tumour necrosis factor. 
2011). Other interesting albeit less replicated findings in schizophrenia show increased levels of IL-1RA and IL-12 (Potvin 2008; Miller 2011). In contrast, a certain degree of inconsistency characterises the findings of other cytokines, such as IL- $1 \beta$, TNF- $\alpha$ or interferon gamma (IFN- $\gamma$ ), which were reported as either increased or at similar levels to those in healthy controls (Potvin 2008; Miller 2011; Zajkowska 2014).

\section{Cytokines and bipolar disorder}

In bipolar disorder, meta-analyses reveal that elevated levels of sIL-2R, IL-4, TNF- $\alpha$, soluble TNF- $\alpha$ receptor 1 (sTNFR1) and soluble IL-6 receptor (sIL-6R) have been consistently reported from the studies conducted so far (Modabbernia 2013; Munkholm 2013). In contrast with what has been reported in depression and schizophrenia, these meta-analyses found no significant differences in IL-6 levels. Findings on IL-1 $\beta$, IL1RA and IL-10 remain inconsistent in bipolar disorder, where either elevated or normal levels have been reported.

\section{Inconsistencies across studies}

The inconsistencies across studies could be partly explained by the great variation in both theoretical and methodological approaches. Indeed, current literature on this topic presents considerable variation in the assessment of inflammatory markers, with regard to both whether serum or plasma samples were used, and to specific analysis techniques. Unfortunately, only a few studies have investigated the role of major confounding factors such as medication, stage of illness, body mass index, smoking status and ethnicity, which may also account for the different findings across studies.

\section{C-reactive protein (CRP)}

C-reactive protein (CRP) is another frequently investigated peripheral inflammatory marker. CRP is an acute-phase protein mainly synthesised by the liver in response to factors released by macrophages and adipocytes. High levels of CRP $(>10 \mathrm{mg} / \mathrm{L})$ are usually interpreted as a sign of infection or systemic inflammation. Interestingly, even a mild elevation in inflammation levels (such as an increase in CRP within a lower range $(>3 \mathrm{mg} / \mathrm{L}))$ is associated with increased risk of cardiovascular disease in apparently healthy individuals. In this context, the high-sensitivity CRP (hsCRP) test has been introduced as an adjunct to traditional risk factor screening for cardiovascular disease (Pearson 2003).

\section{CRP and depression}

To date, the evidence for elevated CRP levels in depression remains inconsistent (Kuo 2005; Howren 2009). The inconsistencies might be partly related to the effect of modifiers, such as body weight as recently suggested by Liu et al (2014). Interestingly, however, a meta-analysis of longitudinal studies measuring CRP shows that increased CRP precedes the onset of depressive symptoms; these findings support a causal role of inflammation in the development of depression (Valkanova 2013). Work partly conducted in our own research group has demonstrated that maltreated children show a significant increase in CRP levels in adulthood (Danese 2007). Interestingly, the effects of childhood maltreatment on CRP appear larger in individuals who become depressed in adulthood (Danese 2008). Childhood maltreatment has been associated not only with depression in adulthood, but also with a variety of other mental and physical illnesses in adults. However, the mechanisms influencing the trajectory towards one or another disorder still remain unknown (Goodwin 2004; Putnam 2013). The immune system is embedded in a network of other biological systems involved in the development of psychopathology (Baumeister 2014), and the dysfunction of this network at different levels could play a role in facilitating progression towards one or another trajectory.

\section{CRP and schizophrenia}

In schizophrenia, CRP levels appear to be reported to be quite consistently elevated. However, the inconsistencies in the association between CRP and factors such as symptoms, antipsychotic treatment and metabolic abnormalities make it difficult to draw conclusions about the specificity of this marker in this condition (Singh 2014). Similar to what has been reported in depression, high CRP levels at the onset of psychosis in adults have been associated with experience of maltreatment in childhood (Hepgul 2012).

\section{CRP and bipolar disorder}

C-reactive protein levels have been also found to be increased in bipolar disorder (Dickerson 2007), during both manic and depressive phases (Dickerson 2007; Cunha 2008; De Berardis 2008; Goldstein 2009). Increased CRP levels have been associated with more severe manic symptoms, and have been suggested as an independent predictor of symptom severity (Dickerson 2007). Moreover, in a more recent study involving people with bipolar disorder, elevated CRP was found to be inversely 
related to cognitive performance (Dickerson 2013). However, interestingly, CRP levels remain significantly elevated during remission phases (Tsai 2012).

\section{Evidence of neuroinflammation in psychiatric disorders}

The evidence from studies on peripheral markers of inflammation has certainly greatly advanced our knowledge and understanding of the role of the immune system in the development of psychiatric disorders. However, how much these peripheral abnormalities reflect central inflammation remains under debate.

At a cellular level, central immune responses are mainly regulated by microglia and astrocytes, which generally play inflammatory and antiinflammatory roles respectively. Microglia are the resident immune cells in the brain and their number and function appear to be tightly controlled by the local microenvironment. Under quiescent conditions, microglia may be involved in facilitation of neurogenesis, but activation of microglia has been implicated in neurogenesis suppression (Yirmiya 2011). Most of the evidence supporting a role of neuroinflammation in the development of psychiatric disorders still comes from preclinical studies. Of course, animal studies conducted in vivo allow direct investigation of the brain during specific treatments (Kreisel 2014). In contrast, human studies are of course limited to either postmortem dissection or in vivo neuroimaging.

To date, only one study has been conducted quantifying microglial activation in vivo in humans (Hannestad 2013). Positron emission tomography (PET) of brain regions of interest in a small sample of depressed individuals and healthy controls showed no significant difference in microglial activation between the two groups. Of note, this study focused only on individuals with mild-to-moderate depression and with low levels of CRP. It might not reflect what is happening in severely depressed patients or depressed patients in whom peripheral inflammation has been reported. A few more PET studies quantifying microglial activation in vivo have been conducted in patients with schizophrenia. These reported increased microglia activation (van Berckel 2008; Doorduin 2009), further supporting the presence of neuroinflammation in this condition. Only one very recent PET study has investigated microglial activation in bipolar disorder, revealing the presence of focal neuroinflammation in the right hippocampus of individuals with the illness (Haarman 2014).
Similarly, very few post-mortem studies have focused on microglia in patients with major depression. These studies actually included patients with different psychiatric diagnoses and could not find a particular effect of diagnosis on density of microglia. They did, however, find an association between suicide and higher microglial density in several brain regions (Steiner 2008). Only recently did Torres-Platas and colleagues (Torres-Platas 2014) provide the first evidence of increased microglial activation in post-mortem brain samples from middle-aged depressed people who died by suicide. Further supporting the presence of a neuroinflammatory process in the same individuals is the high density of perivascular macrophages when compared with matched controls. Those findings are summarised in Box 2.

Post-mortem studies in people with schizophrenia have reported mixed findings, again possibly because of methodological problems: most of these studies have been conducted on relatively small samples, included mainly elderly people in whom incidental lesions are common, and have used different methods for counting astrocytes and microglia (Schnieder 2011). To date, one single study has observed an increase in microglial activation in the post-mortem tissue from the frontal cortex of people with bipolar disorder (Rao 2010).

\section{Are we seeing just a correlational effect?}

Although it remains unclear to what degree inflammatory dysregulation plays a role in the pathogenesis and aetiology of mental illness, and how this may differ between different disorders and syndromes, current evidence suggests more than a correlational effect.

Suggesting a causal effect is the observation that inflammatory probes such as recombinant preparations of interferon alpha (IFN- $\alpha$ ) can elicit a variety of neuropsychiatric symptoms, with differential effects depending on dose and length

BOX 2 Microglia activation and psychiatric disorders

- Findings from in vivo studies point to the presence of microglial activation in schizophrenia

- Most of the evidence supporting microglial activation in depression comes from post-mortem studies

- An association between suicide and higher microglia activation has been reported in studies including patients with different psychiatric disorders 
of treatment. As an acute response, IFN- $\alpha$ can, albeit rarely, induce transient confusional states, generally marked by disorientation, psychomotor retardation, lethargy, psychotic symptoms, Parkinsonism and speech impediments. More long-term effects with ongoing treatment include fatigue and depressive symptoms, which are prevalent in up to $80 \%$ and $60 \%$ of patients respectively. Furthermore, IFN- $\alpha$ treatment may bring on manic symptoms, irritability and hypervigilance in a small proportion of patients (Raison 2005).

\section{Mechanisms linking inflammation to onset of psychiatric disorders}

The mechanisms through which inflammatory cytokines mediate the onset of psychiatric disorders are still largely under investigation and may include interaction with multiple pathways such as monoamine metabolism, neuroendocrine function and synaptic plasticity (Zunszain 2012, 2013). The most exciting data concerning mechanisms underlying the association between inflammation and psychiatric disorders still come from animal studies, where it is possible to investigate different aspects of inflammation directly in the brain tissue. In particular, the above-mentioned study using a chronic unpredictable stress model to induce a depressive condition in rodents has provided direct causal evidence that deviation of microglia from their homeostatic state has an aetiological role in chronic stress-induced depression (Kreisel 2014). These findings advocate microglia as a possible target for further understanding the pathogenesis of depression and for the development of future antidepressant drugs.

As regards the causes of increased inflammation in people with psychiatric disorders, we have briefly mentioned the potential role of stress. However, other possible factors have been considered over the years to explain this observation, such as the presence of an autoimmune activation. Psychiatric disorders have been reported to occur more often among people with autoimmune diseases than in healthy individuals (Iseme 2014). Interestingly, psychiatric research has recently renewed its interest in the role of autoantibodies in the onset of psychiatric disorders. One particular focus of research has been anti- $N$-methyl-D-aspartate (anti-NMDA) receptor encephalitis, which is an autoimmune condition caused by immunoglobulin $\mathrm{G}$ (IgG) antibodies directed against the NMDA glutamate receptor (discussed in Advances by Rickards et al (2014)). About $65 \%$ of people with this condition present with psychiatric symptoms, particularly psychosis. However, only a minority of patients with psychosis test positive for antiNMDA receptor antibodies, and it remains to be established whether this subset of individuals differs from antibody-negative patients in terms of underlying pathology and response to antipsychotic treatment (Pollak 2014).

\section{Clinical relevance and implications for future treatment strategies}

Over the past few years, there has been a lot of discussion about the criteria currently used to classify patients with psychiatric disorders. Indeed, one of the main aspects in which psychiatry differs from other medical specialties is the lack of objective and quantitative (biologically based) diagnostic criteria. As a consequence, we still rely on symptom-based categories when deciding the care of patients with psychiatric disorders, and this is clearly affecting progress in therapeutic development. The recent controversy over the launch of DSM- 5 and the decision of the US National Institute of Mental Health (NIMH) to reorient its research away from DSM categories and support research projects looking across current categories (Davies 2013) clearly show that psychiatry is ripe for a conceptual shift in its understanding of mental disorders. A classification of patients according to biological abnormalities rather than to symptoms would address this challenge, and would considerably help towards developing effective treatments as well as individualising treatments (Cattaneo 2013).

The evidence reported in this article supports a pathophysiological role for inflammation across different psychiatric disorders, at least in a significant subpopulation of patients. This of course suggests possible convergent pathophysiological routes between conditions that have so far been considered separately in diagnostic classifications. Interestingly, although factors such as psychosocial stressors have been increasingly acknowledged to play an important role in the development of various psychiatric disorders, the concept of common biological pathways across conditions remains largely unrecognised. Notably, inflammation can be exacerbated by psychosocial stressors, and could be one of the biological mechanisms mediating the link between stress and onset of these disorders. In the future, inflammation could possibly indicate the development of stressinduced disorder and therefore help a different classification of psychiatric disorders.

This view would, of course, support inflammation as a potential new therapeutic target. Interestingly, a recent clinical trial combining an anti-inflammatory agent with an antidepressant 
in a sample of patients with treatment-resistant depression found an improvement in depressive symptoms when compared with placebo only, in patients with higher CRP levels at baseline (Raison 2013). Thus, identifying the degree of inflammation could be useful for patients who do not respond to psychotropic medications. Inflammation has become clearly recognised as one of the mechanisms mediating the onset of psychiatric disorders. Now further research is needed to clarify which individuals might most benefit from treatment targeting it.

\section{Acknowledgments}

This research has been supported by the National Institute for Health Research (NIHR) Mental Health Biomedical Research Centre at South London and Maudsley NHS Foundation Trust and King's College London. The views expressed are those of the authors and not necessarily those of the NHS, the NIHR or the Department of Health.

\section{References}

Baumeister D, Russell A, Pariante CM, et al (2014) Inflammatory biomarker profiles of mental disorders and their relation to clinical, social and lifestyle factors. Social Psychiatry and Psychiatric Epidemiology, 49: $841-9$.

Cattaneo A, Gennarelli M, Uher R, et al (2013) Candidate genes expression profile associated with antidepressants response in the GENDEP study: differentiating between baseline 'predictors' and longitudinal 'targets'. Neuropsychopharmacology, 38: 377-85

Cunha AB, Andreazza AC, Gomes FA, et al (2008) Investigation of serum high-sensitive $\mathrm{C}$-reactive protein levels across all mood states in bipolar disorder. European Archives of Psychiatry and Clinical Neuroscience, 258: $300-4$.

Danese A, Pariante CM, Caspi A, et al (2007) Childhood maltreatment predicts adult inflammation in a life-course study. Proceedings of the National Academy of Sciences USA, 104: 1319-24.

Danese A, Moffitt TE, Pariante CM, et al (2008) Elevated inflammation levels in depressed adults with a history of childhood maltreatment. Archives of General Psychiatry, 65: 409-15.

Dantzer R, O'Connor JC, Freund GG, et al (2008) From inflammation to sickness and depression: when the immune system subjugates the brain. Nature Reviews Neuroscience, 9: 46-56.

Davies $\mathrm{E}$ (2013) DSM-5 and the rough ride from approval to publication. BMJ, 346: f1918.

De Berardis D, Conti CM, Campanella D, et al (2008) Evaluation of $\mathrm{C}$-reactive protein and total serum cholesterol in adult patients with bipolar disorder. International Journal of Immunopathology and Pharmacology, 21: 319-24.

Di Nicola M, Cattaneo A, Hepgul N, et al (2013) Serum and gene expression profile of cytokines in first-episode psychosis. Brain, Behavior, and Immunity, 31: 90-5.

Dickerson F, Stallings C, Origoni A, et al (2007) Elevated serum levels of $\mathrm{C}$-reactive protein are associated with mania symptoms in outpatients with bipolar disorder. Progress in Neuro-Psychopharmacology and Biological Psychiatry, 31: 952-5.

Dickerson F, Stallings C, Origoni A, et al (2013) Elevated C-reactive protein and cognitive deficits in individuals with bipolar disorder. Journal of Affective Disorders, 150: 456-9.
Doorduin J, de Vries EF, Willemsen AT, et al (2009) Neuroinflammation in schizophrenia-related psychosis: a PET study. Journal of Nuclear Medicine, 50: 1801-7.

Dowlati Y, Herrmann N, Swardfager W, et al (2010) A meta-analysis of cytokines in major depression. Biological Psychiatry, 67: 446-57.

Goldstein BI, Kemp DE, Soczynska JK, et al (2009) Inflammation and the phenomenology, pathophysiology, comorbidity, and treatment of bipolar disorder: a systematic review of the literature. Journal of Clinical Psychiatry, 70: 1078-90.

Goodwin RD, Stein MB (2004) Association between childhood trauma and physical disorders among adults in the United States. Psychological Medicine, 34: 509-20.

Haarman BC, Riemersma-Van der Lek RF, de Groot JC, et al (2014) Neuroinflammation in bipolar disorder - A [(11)C]-(R)-PK11195 positron emission tomography study. Brain, Behavior, and Immunity, 40: 219-25.

Hannestad J, DellaGioia N, Gallezot JD, et al (2013) The neuroinflammation marker translocator protein is not elevated in individuals with mild-tomoderate depression: a [(1)(1)C]PBR28 PET study. Brain, Behavior, and Immunity, 33: 131-8.

Hepgul N, Pariante CM, Dipasquale S, et al (2012) Childhood maltreatment is associated with increased body mass index and increased C-reactive protein levels in first-episode psychosis patients. Psychological Medicine, 42: 1893-901.

Hiles SA, Baker AL, de Malmanche T, et al (2012) A meta-analysis of differences in IL-6 and IL-10 between people with and without depression: exploring the causes of heterogeneity. Brain, Behavior, and Immunity, 26: 1180-8.

Howren MB, Lamkin DM, Suls J (2009) Associations of depression with C-reactive protein, IL-1, and IL-6: a meta-analysis. Psychosomatic Medicine, 71: 171-86.

Iseme RA, McEvoy M, Kelly B, et al (2014) Autoantibodies and depression: evidence for a causal link? Neuroscience and Biobehavioral Reviews, 40 : $62-79$.

Kreisel T, Frank MG, Licht T, et al (2014) Dynamic microglial alterations underlie stress-induced depressive-like behavior and suppressed neurogenesis. Molecular Psychiatry, 19: 699-709.

Kuo HK, Yen CJ, Chang CH, et al (2005) Relation of C-reactive protein to stroke, cognitive disorders, and depression in the general population: systematic review and meta-analysis. Lancet Neurology, 4: 371-80.

Liu Y, Ho RC, Mak A (2012) Interleukin (IL)-6, tumour necrosis factor alpha (TNF-alpha) and soluble interleukin-2 receptors (sIL-2R) are elevated in patients with major depressive disorder: a meta-analysis and metaregression. Journal of Affective Disorders, 139: 230-9.

Liu Y, Al-Sayegh H, Jabrah R, et al (2014) Association between C-reactive protein and depression: modulated by gender and mediated by body weight. Psychiatry Research, 219: 103-8.

Miller BJ, Buckley P, Seabolt W, et al (2011) Meta-analysis of cytokine alterations in schizophrenia: clinical status and antipsychotic effects. Biological Psychiatry, 70: 663-71.

Modabbernia A, Taslimi S, Brietzke E, et al (2013) Cytokine alterations in bipolar disorder: a meta-analysis of 30 studies. Biological Psychiatry, 74: 15-25.

Mondelli V, Cattaneo A, Belvederi Murri M, et al (2011) Stress and inflammation reduce brain-derived neurotrophic factor expression in firstepisode psychosis: a pathway to smaller hippocampal volume. Journal of Clinical Psychiatry, 72: 1677-84.

Munkholm K, Brauner JV, Kessing LV, et al (2013) Cytokines in bipolar disorder vs. healthy control subjects: a systematic review and metaanalysis. Journal of Psychiatric Research, 47: 1119-33.

Pearson TA, Mensah GA, Alexander RW, et al (2003) Markers of inflammation and cardiovascular disease: application to clinical and public health practice. A statement for healthcare professionals from the Centers for Disease Control and Prevention and the American Heart Association. Circulation, 107: 499-511.

Pollak TA, McCormack R, Peakman M, et al (2014) Prevalence of anti$\mathrm{N}$-methyl-D-aspartate (NMDA) receptor antibodies in patients with
MCO answers

$1 d \quad 2$ b 3 c $\quad 4$ e 5 a 
schizophrenia and related psychoses: a systematic review and metaanalysis. Psychological Medicine, 44: 2475-87.

Potvin S, Stip E, Sepehry AA, et al (2008) Inflammatory cytokine alterations in schizophrenia: a systematic quantitative review. Biological Psychiatry, 63: 801-8.

Putnam KT, Harris WW, Putnam FW (2013) Synergistic childhood adversities and complex adult psychopathology. Journal of Traumatic Stress, 26: 435-42.

Raison CL, Demetrashvili M, Capuron L, et al (2005) Neuropsychiatric adverse effects of interferon-alpha: recognition and management. CNS Drugs, 19: 105-23.

Raison CL, Rutherford RE, Woolwine BJ, et al (2013) A randomized controlled trial of the tumor necrosis factor antagonist infliximab for treatment-resistant depression: the role of baseline inflammatory biomarkers. JAMA Psychiatry, 70: 31-41.

Rao JS, Harry GJ, Rapoport SI, et al (2010) Increased excitotoxicity and neuroinflammatory markers in postmortem frontal cortex from bipolar disorder patients. Molecular Psychiatry, 15: 384-92.

Rickards H, Jacob S, Lennox B, et al (2014) Autoimmune encephalitis: a potentially treatable cause of mental disorder. Advances in Psychiatric Treatment, 20: 92-100.

Rohleder N (2014) Stimulation of Systemic Low-Grade Inflammation by Psychosocial Stress. Psychosomatic Medicine.

Schnieder TP, Dwork AJ (2011) Searching for neuropathology: gliosis in schizophrenia. Biological Psychiatry, 69: 134-9.

Singh B, Chaudhuri TK (2014) Role of C-reactive protein in schizophrenia: an overview. Psychiatry Research, 216: 277-85.

Steiner J, Bielau H, Brisch R, et al (2008) Immunological aspects in the neurobiology of suicide: elevated microglial density in schizophrenia and depression is associated with suicide. Journal of Psychiatric Research, 42: 151-7.

Torres-Platas SG, Cruceanu C, Chen GG, et al (2014) Evidence for increased microglial priming and macrophage recruitment in the dorsal anterior cingulate white matter of depressed suicides. Brain, Behavior, and Immunity, 42: 509

Tsai SY, Chung KH, Wu JY, et al (2012) Inflammatory markers and their relationships with leptin and insulin from acute mania to full remission in bipolar disorder. Journal of Affective Disorders, 136: 110-6.

Upthegrove R, Barnes NM (2014) The immune system and schizophrenia: an update for clinicians. Advances in Psychiatric Treatment, 20: 83-91.

Valkanova V, Ebmeier KP, Allan CL (2013) CRP, IL-6 and depression: a systematic review and meta-analysis of longitudinal studies. Journal of Affective Disorders, 150: 736-44.

van Berckel BN, Bossong MG, Boellaard R, et al (2008) Microglia activation in recent-onset schizophrenia: a quantitative (R)-[11C]PK11195 positron emission tomography study. Biological Psychiatry, 64: 820-2.

Yang ZZ, Grote DM, Ziesmer SC, et al (2011) Soluble IL-2Ralpha facilitates IL-2-mediated immune responses and predicts reduced survival in follicular B-cell non-Hodgkin lymphoma. Blood, 118: 2809-20.

Yirmiya R, Goshen I (2011) Immune modulation of learning, memory, neural plasticity and neurogenesis. Brain, Behavior, and Immunity, 25: 181-213.

Zajkowska Z, Mondelli V (2014) First-episode psychosis: an inflammatory state? Neuroimmunomodulation, 21: 102-8.

Zunszain PA Anacker C, Cattaneo A et al (2012) Interleukin-1 beta: a new regulator of the kynurenine pathway affecting human hippocampal neurogenesis. Neuropsychopharmacology, 37: 939-49.

Zunszain PA, Hepgul N, Pariante CM (2013) Inflammation and depression. Current Topics in Behavioral Neurosciences, 14: 135-51.

\section{MCOs}

Select the single best option for each question stem

\section{Cytokines:}

a are mediators of inflammation that can be found only in the periphery of the body and not in the central nervous system

b are synthesised by the liver

c can never cross the blood-brain barrier

$\mathrm{d}$ coordinate immune response both in the periphery of the body and in the brain

e are high molecular weight proteins.

\section{As regards C-reactive protein (CRP):}

a $\mathrm{CRP}$ is mainly produced by the stomach b CRP levels have been found to be increased in bipolar disorder during both manic and depressive phases

c elevated levels of CRP have never been found to be associated with childhood trauma

d levels of CRP $>3 \mathrm{mg} / \mathrm{L}$ are a sign of infection

e elevated levels of CRP have been consistently reported in depression.
3 As regards microglia:

a microglia are immune cells which can be found only in the periphery of the body

b activation of microglia has been reported in vivo in patients with depression

c the number and function of microglia appear to be tightly controlled by local microenvironment in the brain

d post-mortem studies have consistently reported activation of microglia in people who had schizophrenia

e activation of microglia can be studied in vivo in humans using MRI.

4 As regards peripheral inflammatory markers:

a peripheral inflammatory markers are increased in depression and bipolar disorder but not in schizophrenia

b peripheral inflammatory markers are increased in schizophrenia but not in affective disorders

c all pro-inflammatory cytokines are consistently reported to be elevated across all psychiatric disorders d previous studies have consistently found elevated levels of interleukin-2 (IL-2) and soluble IL-2 receptor in depression

e interleukin- 6 has been found to be consistently elevated in depression and schizophrenia but not in bipolar disorder.

5 As regards inflammation:

a inflammatory probes can evoke a variety of neuropsychiatric symptoms

b there is no evidence supporting a pathophysiological role for inflammation in psychiatric disorders

c anti-inflammatory agents administered together with antidepressants improve depressive symptoms regardless of baseline levels of inflammation

$d$ inflammation is the main cause of all psychiatric disorders

e inflammation cannot influence brain activity. 